\title{
Does every bit need the same power? An investigation on unequal power allocation for irregular LDPC codes
}

\author{
Hanghang Qi, Student Member, IEEE, David Malone, Vijay Subramanian \\ Hamilton Institute, National University of Ireland Maynooth, Ireland \\ Email: \{hanghang.qi, david.malone, vijay.subramanian\}@nuim.ie
}

\begin{abstract}
Irregular LDPC codes can have capacityapproaching performance with an iterative BP decoder under AWGN channel with BPSK modulation. Generally in BPSK modulation, every bit within the block has the same power. In this paper, we investigate unequal power allocation (UPA) using BPSK modulation for irregular binary LDPC codes. We show that constant power modulation need not lead to the best performance. With UPA we see gains of up to $0.25 \mathrm{~dB}$, with larger gains possible if the receiver knows the details of the UPA scheme. Optimal power allocation is shown to depend strongly on the codes in use. Our work demonstrates the promise of the UPA scheme for some irregular LDPC codes.
\end{abstract}

\section{INTRODUCTION}

$\mathrm{L}$ ow-density parity-check (LDPC) codes with BPSK modulation have been shown to achieve near-capacity performance with iterative belief propagation (BP) decoding for the AWGN channel. Researchers have shown that irregular LDPC codes have superior performance both theoretically and practically [1-5]. In [5], Chung et al presented a block length $10^{7}$ bit rate-1/2 LDPC code that achieves reliable performance - a $10^{-6}$ bit error rate (BER) - on an additive white Gaussian noise (AWGN) channel with a signal-to-noise ratio (SNR) $E_{b} / N_{0}$ within $0.04 \mathrm{~dB}$ of the Shannon limit. Meanwhile, irregular LDPC codes exhibit natural unequal error protection (UEP) with BP decoding [6-8]. It is widely observed that highly connected nodes are more protected than weakly connected ones. This property is naturally used for UEP information transmission systems such as image transmission, packet transmission and so on [7], [9]. UEP is one of our motivations for studying unequal power allocation.

For binary LDPC codes, in normal BPSK modulation, every bit is modulated into -1 or +1 , with an equal power of 1 . There are other ways to modulate such as soft modulation with unequal power known as unequal power allocation (UPA). In [11] and [12], normal BPSK modulation and BPSK modulation with UPA are analysed. A source-adaptive power allocation scheme is proposed in [13]. UPA schemes have also been applied to image transmission systems, audio transmission systems, multicarrier/OFDM systems and Turbo code systems [14-18].

In this paper, we investigate UPA scheme for irregular LDPC codes, which we believe has received little attention. We give unequal power to different bits within same block of an irregular LDPC code and investigate the code's average performance including BER and block error ratio (BLER) for an AWGN channel. We show that the UPA scheme can improve overall performance and thus give irregular LDPC codes an extra coding gain (we have observed a maximum of $0.25 \mathrm{~dB}$ ). We investigate the best power ratio for the UPA scheme for particular irregular LDPC codes. We find that the average performance is improved whenever the receiver knows that the UPA scheme is applied in the transmitter.

The paper is organized as follows. In Section II, we briefly review the UEP behaviour of irregular LDPC codes. Section III presents our definition of the UPA scheme for LDPC codes including UPA-UI (with updated intrinsic information) and the irregular LDPC codes that we investigate. In Section IV, we determine by simulation the best power ratio of UPA scheme for irregular LDPC codes. In Section V, we present our results of the UPA scheme on irregular LDPC codes, UPA performance when receiver is unaware and aware that UPA is being used, and BER performance with the number of iterations without and with UPA. Finally, we conclude our work and propose possible future work in Section VI.

\section{UNEQUAL PROTECTION OF IRREGULAR LDPC CODES}

In this section, we review the unequal error protection behaviour of irregular binary LDPC codes first. Binary LDPC codes can be described by a bipartite (Tanner) graph. For regular LDPC codes with regular Tanner graph, the degrees of all bit nodes are equal, and the degrees of all check nodes are equal. While for irregular codes, the degrees of nodes on each side can vary widely. In terms of parity-check matrix, $H$, the weight per row and column is not uniform. The degrees are usually chosen according to some distribution.

Within a single block of an irregular LDPC code, some bits are strongly protected and tend to have a lower BER while other bits are less protected and tend to have a higher BER. This is usually related to the degree distribution and connectivity of bit nodes of the Tanner graph of the LDPC codes. We give a simple example here. Consider a code with block length $N=155$ and coding rate approximately 0.4 where the first 62 bits (group 1) have degree 3, the last 93 bits (group 2) have degree 2 (the exact construction is described later). The average bit error rate for group 1 is denoted BER1, for group 2 is BER2 and the average is BER. The UEP performance of the 155 irregular LDPC codes with BPSK under AWGN channel is shown in Fig. 1. For the two groups of bits in the 155 codes, at the BER level of $10^{-4}$, there is already over $0.2 \mathrm{~dB}$ of difference. Note that all the simulations in this paper are run until 50 block errors happen for each point. 


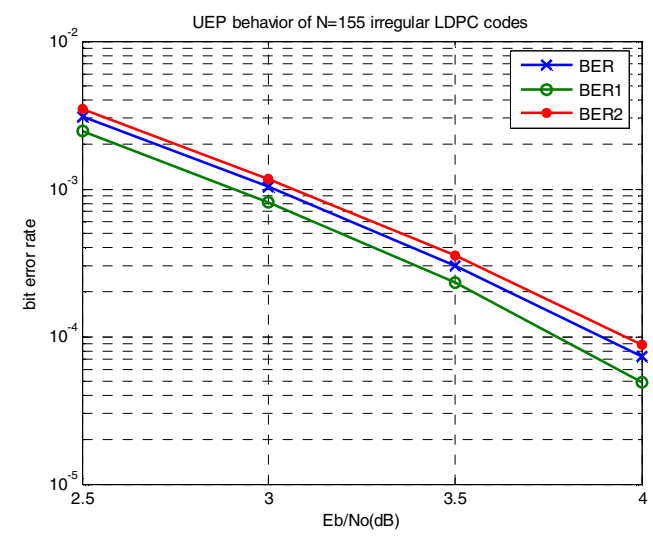

Fig. 1 UEP behaviour of $\mathrm{N}=155$ irregular LDPC codes.

\section{UNEQUAL POWER ALLOCATION ON IRREGULAR LDPC}

\section{A. Unequal power allocation scheme}

Our UPA scheme on LDPC codes is constructed such that different bits can have different modulation power with an average power constant while still using BPSK modulation. On a code of block size $\boldsymbol{N}$, we define $\boldsymbol{P}(\boldsymbol{i})$ as the power used for bit $\boldsymbol{i}$. We require the mean power constraint,

$$
\frac{1}{N}{ }_{i=1}^{N} P(i)=1,
$$

to hold, though $\boldsymbol{P}(\boldsymbol{i})$ is not necessarily constant for every $\boldsymbol{i}$.

In particular, we investigate irregular LDPC codes with two groups of bits. To the $\boldsymbol{N}_{1}$ bits in group 1 we allocate power $\boldsymbol{P}_{1}$, and $\boldsymbol{N}_{2}$ bits in group 2 we allocate $\boldsymbol{P}_{2}$. The constraint is

$$
\frac{N_{1}}{N} \boldsymbol{P}_{1}+\frac{N_{2}}{N} \boldsymbol{P}_{2}=1,
$$

so the average power is still 1 . Given a desired power ratio, $\boldsymbol{a}$ $=\boldsymbol{P}_{\boldsymbol{2}} / \boldsymbol{P}_{2}$, we may solve for $\boldsymbol{P}_{\boldsymbol{1}}$ and $\boldsymbol{P}_{2}$ and then set the BPSK modulation amplitude accordingly.

\section{B. Receiver with and without knowledge of UPA}

We investigate the performance of UPA scheme on irregular LDPC codes both when the receiver knows UPA is used and when it does not know about UPA. We use a BP decoder with maximum 30 iterations. In the decoder for LDPC codes, intrinsic information from the channel is used, which includes the modulation power and the variance of the AWGN channel.

In a UPA BPSK modulation system for an AWGN channel, $N\left(0, \sigma^{2}\right)$, let $x^{\prime}=\left\{x^{\prime}{ }_{1}, x^{\prime}{ }_{2}, \ldots x^{\prime}{ }_{N}\right\}$ denote the codeword. $x=\left\{x_{1}\right.$, $\left.\mathrm{x}_{2}, \ldots \mathrm{x}_{\mathrm{N}}\right\}$ is the modulated vector $\left(0->+\sqrt{P_{i}}, 1->-\sqrt{P_{i}}\right)$. For normal BPSK $P_{i}$ is 1 for all $i$. $\mathrm{y}=\left\{\mathrm{y}_{1}, \mathrm{y}_{2}, \ldots \mathrm{y}_{\mathrm{N}}\right\}$ is the vector after channel. As the channel is AWGN, $y_{i}=x_{i}+n_{i}$, where $n_{i}$ is an i.i.d. $N\left(0, \sigma^{2}\right)$.

The soft value $\boldsymbol{f}_{\boldsymbol{i}}$ of bit $\boldsymbol{i}$ in a $\log$-likelihood ratio domain BP decoder is calculated as follows.

$$
\begin{gathered}
f_{i}=\log \frac{P\left(x_{i}^{\prime}=0 \mid y_{i}\right)}{P\left(x_{i}^{\prime}=1 \mid y_{i}\right)}=\log \frac{P\left(x_{i}=+\sqrt{P_{i}} \mid y_{i}\right)}{P\left(x_{i}=-\sqrt{P_{i}} \mid y_{i}\right)} \\
=\log \frac{P\left(y_{i} \mid x_{i}=+\sqrt{P_{i}}\right)}{P\left(y_{i} \mid x_{i}=-\sqrt{P_{i}}\right)}=\log \frac{P\left(n_{i}=y_{i}-\sqrt{P_{i}}\right)}{P\left(n_{i}=y_{i}+\sqrt{P_{i}}\right)} \\
=\log \frac{\frac{1}{\sqrt{2 \pi} \sigma} e^{-\frac{\left(y_{i}-\sqrt{P_{i}}\right)^{2}}{2 \sigma^{2}}}}{\frac{1}{\sqrt{2 \pi} \sigma} e^{-\frac{\left(y_{i}+\sqrt{P_{i}}\right)^{2}}{2 \sigma^{2}}}}=2 y_{i} \sqrt{P_{i}} / \sigma^{2}
\end{gathered}
$$

When the receiver knows that UPA scheme is used, it will use the known $\boldsymbol{P}_{\boldsymbol{i}}$, otherwise it will suppose $\boldsymbol{P}_{\boldsymbol{i}}=1$ and will just use $2 y_{i} / \sigma^{2}$ as the intrinsic information instead. The UPA scheme can thus be viewed as providing an unequal intrinsic information distribution as well.

In later sections, we show the performance of UPA in both cases, where the updated intrinsic information is and is not adapted for UPA system. We name the schemes as UPA and UPA-UI (updated intrinsic) respectively. UPA scheme shows improved performance in both situations though performance is better when the accurate intrinsic information is used.

\section{Irregular LDPC codes construction}

The irregular LDPC codes that we use to investigate the UPA Scheme are the codes punctured from Quasi-cyclic (QC) LDPC codes based on circulant matrices [10]. The reason that we chose QC LDPC codes is because the regular codes are strong regular LDPC codes. Every bit in the block has exactly the same bit error ratio. They also achieve a good trade-off between encoder complexity and decoding performance. We then modify the regular LDPC codes to be irregular by puncturing some sub-matrices with zero matrices. The irregular codes that we get have two groups of bits with different error protection ability. For example, using length 155 codes, the parity-check matrix becomes:

$$
H=\left[\begin{array}{ccccc}
I_{1} & I_{2} & I_{4} & I_{8} & 0 \\
I_{5} & I_{10} & I_{20} & 0 & I_{18} \\
I_{25} & I_{19} & 0 & I_{14} & I_{28}
\end{array}\right]_{(93 \times 155)}
$$

The other codes have similar construction and structure. All the irregular codes that we investigated are listed in Table 1.

Table 1. Irregular LDPC codes investigated in this paper.

\begin{tabular}{|c|c|c|c|}
\hline \hline $\begin{array}{c}\text { Block } \\
\text { size }\end{array}$ & $\begin{array}{c}\text { Code } \\
\text { rate }\end{array}$ & $\begin{array}{c}\text { Original regular codes } \\
\text { (column degree, row } \\
\text { degree) }\end{array}$ & $\begin{array}{c}\text { Modified } \\
\text { Irregular codes }\end{array}$ \\
\hline 155 & 0.4129 & $(3,5)$ & $\begin{array}{c}2 / 5 \text { bits (group 1) degree 3 } \\
3 / 5 \text { bits (group 2) degree 2 }\end{array}$ \\
\hline 305 & 0.4065 & $(3,5)$ & $\begin{array}{c}2 / 5 \text { bits (group 1) degree 3 } \\
3 / 5 \text { bits (group 2) degree 2 }\end{array}$ \\
\hline 905 & 0.4022 & $(3,5)$ & $\begin{array}{c}3 / 5 \text { bits (group 1) degree 3 } \\
2 / 5 \text { bits (group 2) degree 2 }\end{array}$ \\
\hline 1655 & 0.4005 & $(3,5)$ & $\begin{array}{c}3 / 5 \text { bits (group 1) degree 3 } \\
2 / 5 \text { bits (group 2) degree 2 }\end{array}$ \\
\hline 1928 & 0.3771 & $(5,8)$ & $\begin{array}{c}3 / 8 \text { bits (group 1) degree 5 } \\
5 / 8 \text { bits (group 2) degree 3 }\end{array}$ \\
\hline
\end{tabular}




\begin{tabular}{|c|c|c|c|}
\hline 2248 & 0.3768 & $(5,8)$ & $\begin{array}{l}3 / 8 \text { bits (group 1) degree 5 } \\
5 / 8 \text { bits (group 2) degree 3 }\end{array}$ \\
\hline 2947 & 0.4296 & $(4,7)$ & $\begin{array}{l}3 / 7 \text { bits (group 1) degree } 4 \\
4 / 7 \text { bits (group 2) degree 3 }\end{array}$ \\
\hline
\end{tabular}

\section{Power Allocation Ratio, UEP AND BER}

We now investigate the UPA scheme on irregular LDPC codes. We use UPA-UI with a BP decoder with maximum 30 iterations. For each fixed average SNR we determine the performance of the UPA scheme for each LDPC codes by change different power allocation ratio $\boldsymbol{a}$, and monitoring BER1, BER2, BER and BLER. The results for each code on Table 1 are shown in Fig. 3 to Fig. 8 respectively.

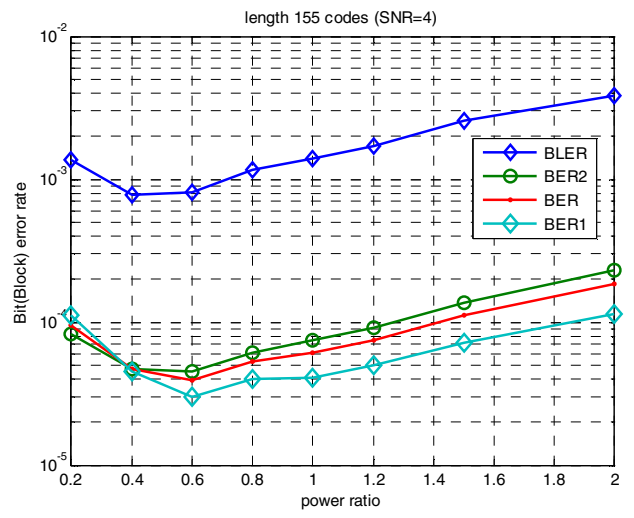

Fig. 3 Length 155 codes UPA-UI performance vs. power ratio $(\mathrm{SNR}=4)$.

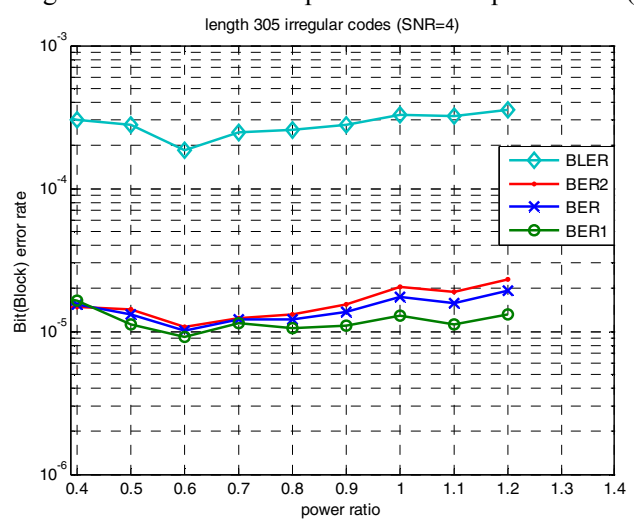

Fig. 4 Length 305 codes UPA-UI performance vs. power ratio ( $S N R=4)$.

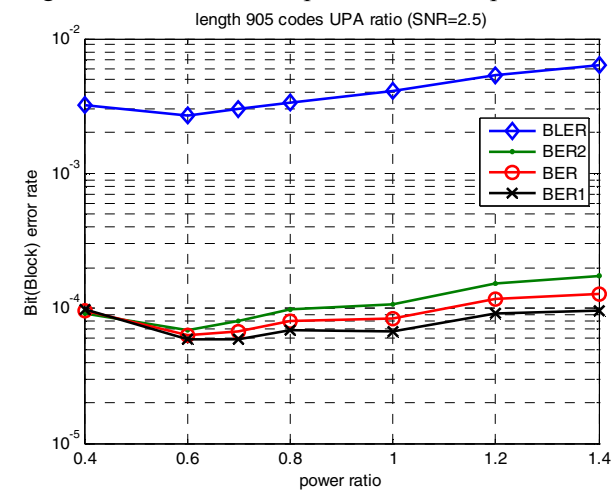

Fig. 5 Length 905 codes UPA-UI performance vs. power ratio $(\mathrm{SNR}=2.5)$.

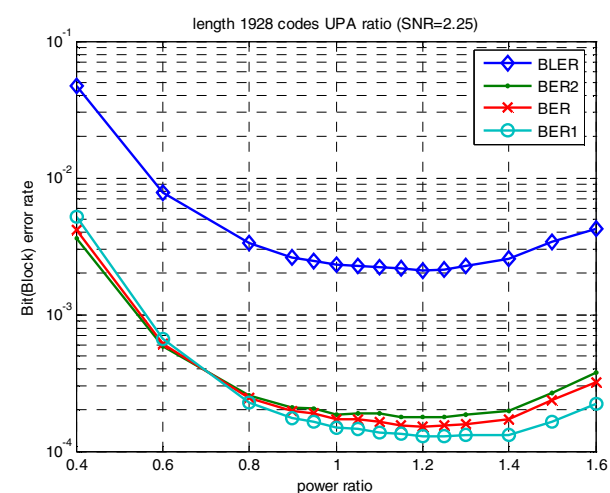

Fig. 6 Length 1928 codes UPA-UI performance vs. power ratio ( $\mathrm{SNR}=2.25)$.

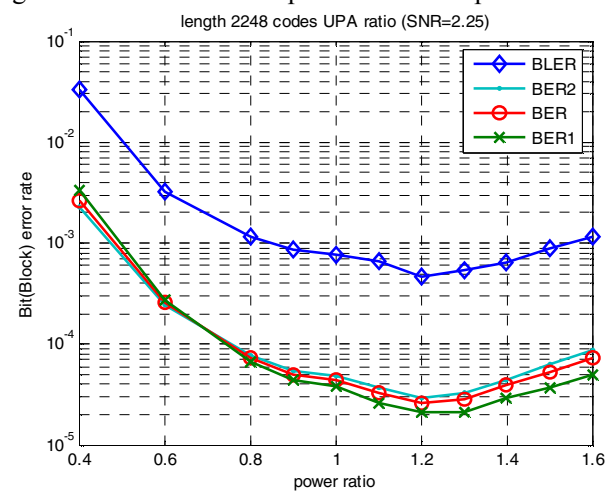

Fig. 7 Length 2248 codes UPA-UI performance vs. power ratio ( $\mathrm{SNR}=2.25)$.

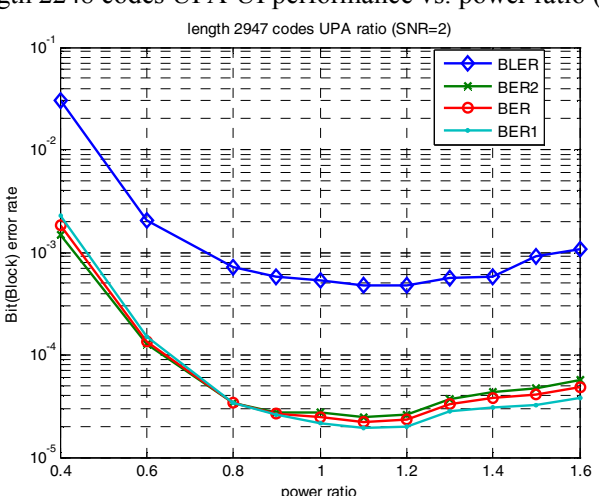

Fig. 8 Length 2947 codes UPA-UI performance vs. power ratio ( $\mathrm{SNR}=2$ ).

The results clearly show that BER and BLER change considerably when the power ratio $\boldsymbol{a}$ changes. The minimum of BER and BLER does not necessarily occur at $\boldsymbol{a}=1$ (i.e. normal BPSK). For the codes with length 155, 305, 905 and 1655 , the optimum power ratio is smaller than $1,(0.6,0.6,0.7$, 0.7 respectively), while for the codes with length 1928,2249 and 2947 , the power ratio for best average performance is slightly larger than 1 . We note that there seems to be less separation between the BER1 and BER2 for the longer codes that we use here, and the BLER curve is quite flat above 1. This suggests that the optimal power ratio is linked to the code structure.

Not surprisingly, we observe that the UEP behaviour of BER1 and BER2 changes with the power ratio changes. BER1 
is smaller than BER2 for all of our 7 codes when power ratio is 1 (normal BPSK). When power ratio becomes small, BER1 becomes close to BER2 and at some point they become equal and then BER2 becomes smaller than BER1. But the power ratio of the change-over point is not necessarily the optimal power ratio for BER and BLER. It is also surprising that sometimes we give less power to less protected bits.

It is also interesting to note that short and medium irregular LDPC codes have a larger range of power ratios, which keep BER and BLER performance at a similar level, but long codes have more abrupt changes in the performance-power ratio curve. The performance drops faster for 2248 and 2947 codes when power ratio goes away from 1 .

\section{Performance of UPA on IRREgUlar LDPC codes}

\section{A. Performance of UPA on irregular LDPC codes}

Having demonstrated the promise of UPA, we now look at performance gains over a range of SNRs and the impact of accurate intrinsic information. The UPA and UPA-UI schemes use close optimal powers found from the previous section (we note it is possible that the optimal power ratio is different for UPA and UPA-UI). Results are shown in Fig. 9 to Fig. 13. All the results show better average performance for UPA over an equal power allocation and performance improves further when the intrinsic information is updated.

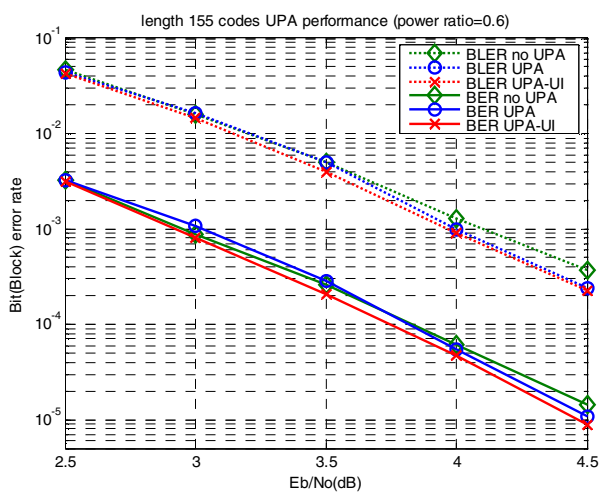

Fig. 9 Comparison of schemes on length 155 irregular LDPC codes.

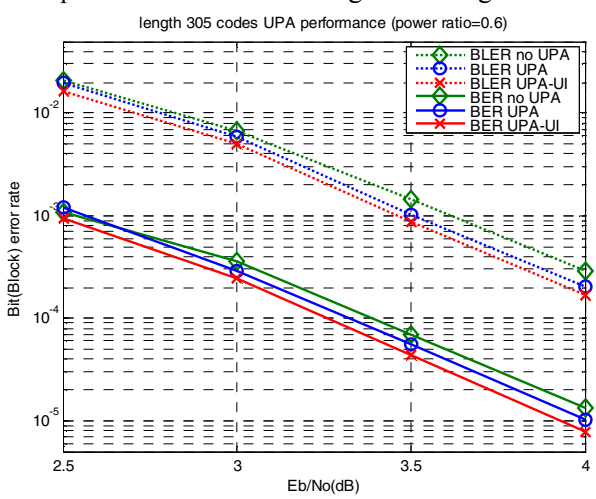

Fig. 10 Comparison of schemes on length 305 irregular LDPC codes.

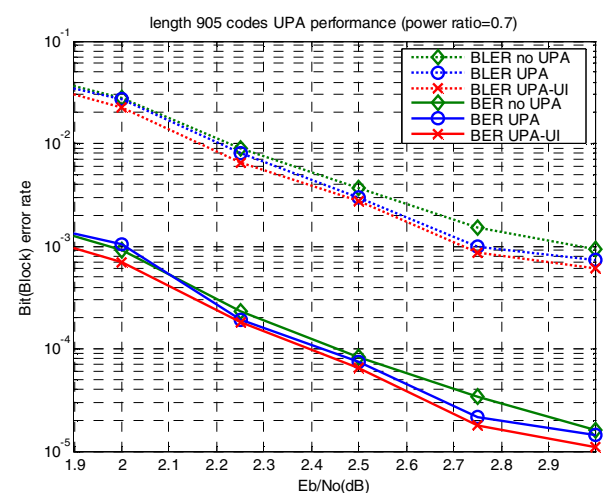

Fig. 11 Comparison of schemes on length 905 irregular LDPC codes.

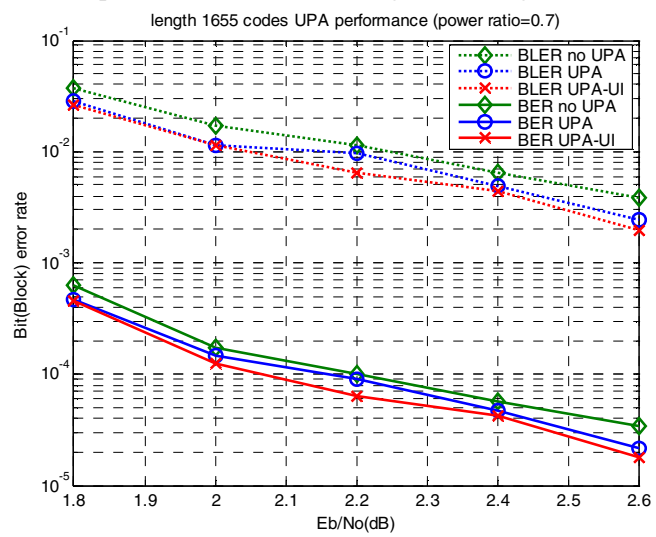

Fig. 12 Comparison of schemes on length 1655 irregular LDPC codes.

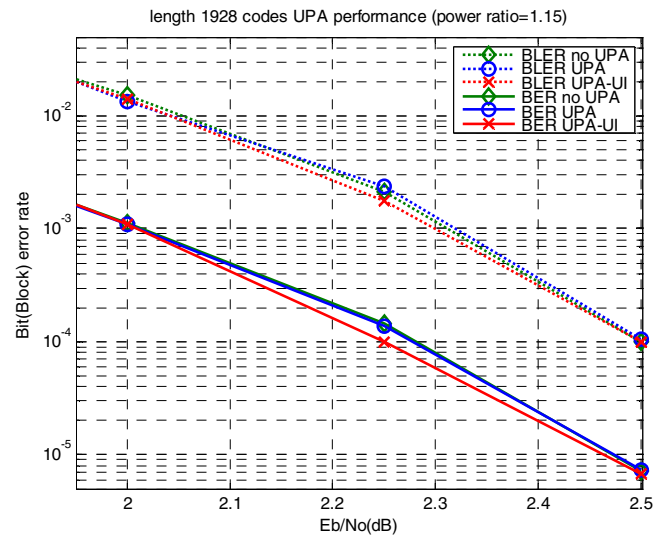

Fig. 13 Comparison of schemes on length 1928 irregular LDPC codes.

With the UPA-UI scheme, at the BER at around $10^{-5}$, there is a $0.2 \mathrm{~dB}$ gain for 155 codes, $0.2 \mathrm{~dB}$ gain for 305 codes, and $0.25 \mathrm{~dB}$ gain for 905 codes and 1655 codes. For other codes, the gain is smaller. This could because of the structure of these codes, their UEP behaviour is not dominant, as the optimum power ratio is very close to 1 .

\section{B. BER Performance of UPA vs. iterations}

Figure 14 shows how BER performance improves as the number of BP iterations increases without and with UPA-UI. 
We see similar performance up to 10 iterations, but the real advantage of UPA becomes apparent later. We have seen broadly similar results for other codes and SNR values. This suggests that the gains from UPA are genuinely being incorporated by the decoder and are not a simple first order effect. Beyond 10 iterations, UPA shows an advantage over the scheme without UPA. This suggests that UPA offers gains even for relatively small number of iterations.

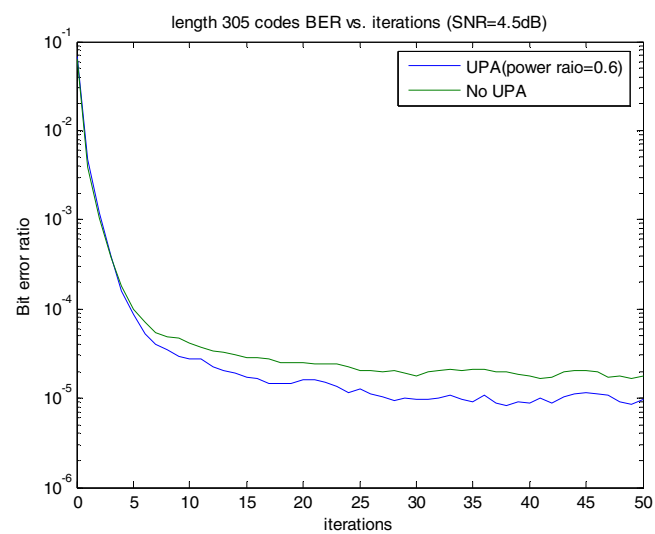

Fig. 14 BER vs. iterations of length 305 irregular LDPC codes.

\section{CONCLUSIONS AND FURTHER WORK}

In this paper, we investigated an unequal power allocation (UPA) modulation scheme for irregular LDPC codes under an AWGN channel. We found that a power ratio of 1 is not necessarily optimal and UPA can give irregular LDPC codes an extra performance gain. The largest gain was $0.25 \mathrm{~dB}$ in our simulations. We studied the codes performance with different power ratios. The optimum power ratio can be smaller or larger than 1 and does not seem to allocate similar BERs to both groups of bits. The reason for this is not clear, but we speculate that it relates to the structure of the codes. We also studied the behaviour of UPA when the receiver uses the power allocation as part of its intrinsic information. We found this further improves the performance of UPA. BER performance vs. decoding iterations is also investigated and it is shown that UPA can improve the BER performance in early stages (around 10 iterations) of decoding.

As future work we hope to investigate more general irregular LDPC codes with UPA beyond codes with 2 groups of bits. The relationship between optimal power allocation and code structure is also an interesting one. For an irregular code, an analytical scheme for finding the best power allocation would be desirable.

\section{REFERENCES}

[1] M. Luby, M. Mitzenmacher, A. Shokrollahi, and D. Spielman, "Improved Low-density parity-check codes using irregular graphs", IEEE Trans. Inform. Theory, vol. 47, $585-598$, Feb. 2001
[2] M. Luby, M. Mitzenmacher, A. Shokrollahi, and D. Spielman, "Efficient Erasure Correcting Codes", IEEE Trans. Inform. Theory, vol. 47, 569584, Feb. 2001.

[3] T.J. Richardson and R. Urbanke "Design of capacity-approaching irregular low-density parity-check codes", IEEE Trans. Inform. Theory, vol. 47, 619 -637 , Feb. 2001

[4] D. MacKay, S. Wilson, and M. Davey, "Comparison of constructions of irregular Gallager codes", IEEE Trans. Commun., vol. 47, 1449-1454, Oct. 1999

[5] S.-Y. Chung, G. D. Forney, Jr., T. J. Richardson, and R. Urbanke, "On the design of low-density parity-check codes within $0.0045 \mathrm{~dB}$ of the Shannon limit," IEEE Commun. Lett., vol. 5, 58-60, Feb. 2001.

[6] C. Poulliat, D. Declercq, and I. Fijalkow, "Enhancement of Unequal Error Protection Properties of LDPC Codes", EURASIP Journal on Wireless Communications and Networking, Volume 2007.

[7] G. Al-Regib, Y. Altunbasak, "An unequal error protection method for packet loss resilient 3D mesh transmission”, INFOCOM 2002. Vol 2, Issue, 2002, 743-752.

[8] X. Yang, D. Yuan, P. Ma, and M. Jiang, "New research on unequal error protection (UEP) property of irregular LDPC codes," in Proceedings of IEEE Consumer Communications and Networking Conference (CCNC '04), pp. 361-363, Las Vegas, Nev, USA, January 2004.

[9] X. Gao, H. Zhang, D. Yuan, "Unequal Error Protected Image Transmission over Multilevel coded Wavelet Packet Multicarrier Modulation System", ISIT 2004. Proceedings. International Symposium on info. Theory, 2004, $390-390$

[10] R. M. Tanner, D. Sridhara, A. Sridharan, T. E. Fuja, and D. J. Costello, "LDPC block and convolutional codes based on circulant matrices", IEEE Trans. on Information Theory, vol. 50, no. 12, 2966-2984, Dec. 2004.

[11] T. Briggen and P. Vary, "Analysis of Modulation with Unequal Power Allocation", in Proc. of IEEE VTC Spring, Sweden, May 2005.

[12] T. Bruggen, M. Adrat and P. Vary, "BPSK modulation with unequal power allocation considering a priori knowledge", IEEE 16th International Symposium on Personal, Indoor and Mobile Radio Communications (PIMRC 2005). Vol. 2, Sept. 2005, $1071-1075$.

[13] N. Goertz and E. Bresch, "Source-Adaptive Power Allocation for Digital Modulation,” IEEE Comm. Lett. Vol. 7, 569-571, Dec 2003.

[14] M.F. Sabir, R.W. Heath, A.C. Bovik, "Unequal Power Allocation for JPEG Transmission over MIMO Systems", Asilomar Conference on Signals, Systems and Computers Oct/Nov 2005, 1608-1612

[15] Z. Ahmad, S. Worrall, A. Kondoz, "Unequal power allocation for scalable video transmission over WiMAX", IEEE International Conference on Multimedia and Expo, 2008, 517-520.

[16] Keang-Po Ho, "Unequal Error Protection Based on OFDM and its application in digital audio transmission", Global Telecommunications Conference (GLOBECOM 98). Vol 3, Nov 1998, 1320-1325.

[17] B. Balamuralithara, "A New Unequal Power Allocation Scheme for Asymmetric Turbo Code System", Wireless Personal Communications, Jun 2008.

[18] A.H.S. Mohammadi, A.K. Khandani, "Unequal power allocation to the turbo-encoder output bits with application to CDMA systems", IEEE Trans. on Communications, Nov 1999, Vol. 47, Issue 11, 1609-1610 